IP Periodica Polytechnica Chemical Engineering

60(2), pp. 69-73, 2016

DOI: $10.3311 /$ PPch. 8363

Creative Commons Attribution (i)

RESEARCH ARTICLE

\section{Applicability of Ultra Performance Convergence Chromatography, a New Generation of Supercritical Fluid Chromatography, for the Analysis of Pesticide Residues}

\author{
Andrea Vass ${ }^{1 *}$, Barnabás Attila Rácz ${ }^{1,2}$, László Abrankó ${ }^{1}$
}

Received 26 June 2015; accepted after revision 28 September 2015

\begin{abstract}
Monitoring and controlling wide variety of pesticide residues is a crucial challenge of food safety. In our study ultra-performance convergent chromatography (UPC $\left.{ }^{2}\right)$, as the new generation of supercritical fluid chromatography coupled with ESI-MS/MS system was applied to separate a set of pesticides to investigate their chromatographic behavior under various $U P C^{2}$ conditions. 30 components were selected representing the GC and LC measurable components. Capacity factors obtained from LC and GC runs UPC'-PDA were compared. Based on our data UPC ${ }^{2}$ should be considered as an alternative chromatographic approach with separation mechanisms not yet fully characterized. Interestingly the type of mobile phase modifier influences the ionization in an ESI-MS system.
\end{abstract}

\section{Keywords}

ACQUITY UPC $C^{2}$ Pesticides, LC-GC, SFC

\footnotetext{
${ }^{1}$ Department of Applied Chemistry, Faculty of Food Science, Corvinus University of Budapest, Villányi út 29-43, H-1118, Budapest, Hungary

${ }^{2}$ Faculty of Chemical Technology and Biotechnology, Budapest University of Technology and Economics, Múegyetem rkp. 3. H-1111, Budapest, Hungary

${ }^{*}$ Corresponding author, e-mail: Vass.Andrea@etk.szie.hu
}

\section{Introduction}

From the middle of the $20^{\text {th }}$ century the increased industrialization of food processing and agriculture made pesticides more frequent in our life. Treating crops with pesticides is believed as a proper tool to maintain stable supply of agricultural products to serve the needs of the increasing world population. However by applying pesticides the number of food safety issues is also raised therefore the registration and the monitoring of these chemicals are inevitable. More than 300 pesticides are registered nowadays. [1]

These days pesticides analysis is preferably carried out by using GC or LC combined with mass spectrometry, predominantly with MS/MS or TOFMS [2, 3]. In some cases detection is based on UV. [4] The selection of separation method depends on the volatility and the thermostability of the target components. If targeted analytes are sufficiently volatile, or can be converted to volatile derivate with chemical modification, without decomposition, GC is the preferred method for separation due to the higher achievable chromatographic resolution. If the analyte contains more polar and/or thermally unstable compounds, the application of LC separation is the appropriate choice [5].

Supercritical fluid chromatography (SFC) as an alternative chromatographic approach might also have feasibility in pesticide analyses. However the application of SFC in this field was highly ignored until these days and only limited literature is available [7]. Probably this is mainly due to hardwarerelated technical limitations, among which the insufficient reproducibility of former SFC instrumentation was considered the most severe one [6]. Recently appeared SFC hardware solutions from Agilent Technologies called ultraperformance supercritical fluid chromatography (UHSFC) and by Waters Company called ultra-performance convergent chromatography $\left(\mathrm{UPC}^{2}\right)$ are advertised attributed to be able to achieve fast and easily reproducible results. Additionally since $\mathrm{CO}_{2}$ (as the main mobile phase) can easily and reproducibly be modified with numerous co-eluents, e.g., hexane, acetonitrile, methanol, ethyl acetate etc., it is claimed that either GC-like and/or LC-like conditions can be similarly set in this system [8]. 
In this study we challenged convergence chromatographic separation to a carefully selected set of analytes that similarly contained representatives of those compound-types, which are typically considered as GC or LC amenable ones [9]. Our goal was to investigate some basic chromatographic properties (capacity factor and peak shape) of these selected pesticides and comparing the chromatographic results of LC and GC runs related to the same compound.

\section{Materials and methods}

\subsection{Reagents}

HPLC-grade acetonitrile $\left(\mathrm{CH}_{3} \mathrm{CN}\right)$, methanol $\left(\mathrm{CH}_{3} \mathrm{OH}\right)$ and formic acid $(\mathrm{HCOOH})$ were delivered by VWR; Radnor, PA, USA. $\mathrm{CO}_{2}$ was obtained from Merck (Darmstadt, Germany). The standards (aldrin, azinphos-methyl, azoxystrobin, boscalid, brompropylate, carbendazim, chlorpropham, diazinon, dichloran, dichlorvos, dimethoate, esfenvalerate, fenhexamid, flutriafol, folpet, heptachlor, pendimethalin, permethrin, piperonyl-butoxide, pirimicarb, pirimiphos-methyl, prochloraz, quintozene, spiroxamine, tau-fluvalinate, tebufenpyrad, thiabendazole, triadimenol, trifloxystrobin, ó-endosulfan.) were obtained from Sigma-Aldrich (Schnelldorf, Germany), Milli-Q-Plus ultra-pure water system from Millipore (MerckMillipore, Milford, MA, USA) was used throughout the study.

Stock solutions were prepared by dissolving an accurately weighted portion of the pesticides (approximately $10 \mathrm{mg}$ powder or liquid) in $5 \mathrm{ml}$ of an appropriate solvent, and they were stored at $-18{ }^{\circ} \mathrm{C}$. Working mixtures $\left(10.0 \mu \mathrm{g} \mathrm{mL} \mathrm{m}^{-1}\right)$ were obtained by further dilution with $\mathrm{CH}_{3} \mathrm{CN}$ and $\mathrm{CH}_{3} \mathrm{OH}$ individually to the UPC2 PDA detection. The selection of appropriate solvents based on the type of the applied co-solvent. Using the single compound working solutions, two $0.6 \mu \mathrm{g} \mathrm{mL}^{-1}$ multicompound mixtures were prepared. Analytes were split into two mixture solutions to exclude the simultaneous presence of isobaric compounds (e.g., fenhexamid $(\mathrm{M}+\mathrm{H}+: 302.0)$ and flutriafol $(\mathrm{M}+\mathrm{H}+: 302.1))$ in the same mixture. Both mixtures contained also common compounds such as Boscalid and Piperonyl-butoxide. Two working mixtures were prepared twice either in $\mathrm{CH}_{3} \mathrm{CN}$ or $\mathrm{CH}_{3} \mathrm{OH}$ for different $\mathrm{UPC}^{2}-\mathrm{MS}$ experiments. In the LC-ESI-MS experiments, mixtures were dissolved in $80: 20 \mathrm{v} / \mathrm{v} \%$ water and the appropriate mobile phase solvent $\mathrm{CH}_{3} \mathrm{CN}$ and $\mathrm{CH}_{3} \mathrm{OH}$ were used.

\subsection{Instrumentation}

\subsubsection{UPC²-PDA System}

The chromatographic measurement was performed using ACQUITY UPC ${ }^{2}$ system (UPC ${ }^{2}$ ) (Waters, Milford, MA, USA) with binary solvent manager, sample manager, column manager convergence chromatography manager and PDA (Photodiode Array Detector) detector. The separations were carried out via Acquity $\mathrm{UPC}^{2} \mathrm{C} 18$ column $(3 \mathrm{~mm} \times 100 \mathrm{~mm}, 1.8 \mu \mathrm{m})$, the mobile phase was $\mathrm{CO}_{2}\left(99.97 \%\right.$ purity) and $\mathrm{CH}_{3} \mathrm{OH}$ or $\mathrm{CH}_{3} \mathrm{CN}$ were used as co-eluents (CoElu). The mobile phase gradient was the following: $0-1 \mathrm{~min}: 3 \% \mathrm{CoElu}, 1-9 \mathrm{~min}$ : $20 \% \mathrm{CoElu}$, 9-9.5: 20 to $3 \%$ CoElu, $9.5-13 \mathrm{~min}$ : $3 \%$ CoElu at a flow rate of $1.5 \mathrm{~mL} \mathrm{~min}^{-1}$. In the case of $\mathrm{CH}_{3} \mathrm{CN}$ the post run was kept until $14.5 \mathrm{~min}$. The injected sample volume was $5 \mu \mathrm{l}$. The back pressure of the system was 1500 psi and column temperature was set on $40,50,60$ and $70{ }^{\circ} \mathrm{C}$. The detection was performed with PDA, between 210-400 nm wavelength.

\subsubsection{UPC ${ }^{2}$-ESI-MS}

QTRAP 3200 triple quadrupole-linear ion trap mass spectrometer (Applied Biosystems/Sciex, Foster City, CA, USA) was applied as detection system. The instrument was equipped with a Turbo V interface and Turbo Ion Spray probe (Applied Biosystems), operating in positive ion mode. The UPC ${ }^{2}$-ESIMS was coupled with an Agilent 1100 HPLC system (Agilent Technologies, Waldbronn, Germany) $\mathrm{UPC}^{2}$ flow rate $1.5 \mathrm{~mL} \mathrm{~min}^{-1}$ and the HPLC flow rate $0.2 \mathrm{~mL} \mathrm{~min}^{-1}$ passed thought the splitter and the mixture was introduced to the ESI. The HPLC pump carried 50:50 v/v\% $\mathrm{CH}_{3} \mathrm{CN}$ : water with $0.1 \mathrm{v} / \mathrm{v} \% \mathrm{HCOOH}$. Since the controlling of HPLC-ESI-MS and $\mathrm{UPC}^{2}$ systems with one PC was unsolvable, the systems were driven by two separate computers.

The UPC ${ }^{2}$-ESI-MS coupled system was able to work only with manual injection. The gradient was the same described before. The back pressure of the system was 1500 psi. This value was set after the coupling and applied to the UPC ${ }^{2}$-PDA method because by MS coupling the back pressure regulator was unable to keep neither 3000 nor 2000 psi pressure previously tried to set. Figure 1 shows the UPC ${ }^{2}$-ESI-MS system.

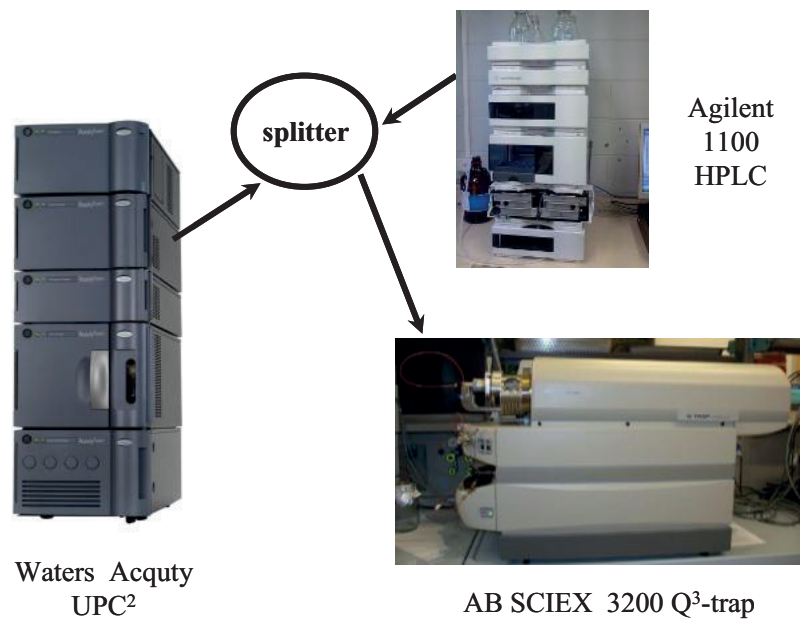

Fig. 1 Applied UPC²-ESI-MS system

\subsubsection{HPLC-ESI-MS System}

The above described QTRAP 3200 triple quadrupole-linear ion trap mass spectrometer (Applied Biosystems/Sciex; Foster City, CA, USA) was coupled with an Agilent 1100 HPLC system (Agilent Technologies, Santa Clara, CA, USA) by XBridge TM C18 column $(3.5 \mathrm{~mm} \times 100 \mathrm{~mm} \times 3.5 \mu \mathrm{m}$; Agilent $)$ using 
with gradient elution. The eluent consisted of water containing $0.1 \mathrm{v} / \mathrm{v} \% \mathrm{HCOOH}$ (eluent $\mathrm{A}$ ) and $\mathrm{CH}_{3} \mathrm{CN}$ or $\mathrm{CH}_{3} \mathrm{OH}$ (eluent $\mathrm{B}$ ). Gradient elution was set as follows: $0-1 \mathrm{~min} 5 \% \mathrm{~B} ; 1-30 \mathrm{~min}$ up to $100 \% \mathrm{~B}$; $30-35 \mathrm{~min} 100 \% \mathrm{~B}$; followed by 5 -min equilibration at $5 \% \mathrm{~B}$. The flow-rate was $0.3 \mathrm{ml} \mathrm{min}{ }^{-1}$ while the injection volume was $5 \mu \mathrm{l}$. The column temperature was set to $25^{\circ} \mathrm{C}$. The optimum settings of the HPLC-ESI-MS/MS coupling were as follows: ion spray voltage: $5500 \mathrm{~V}$; curtain gas (N2): 10 psi; ion source gas: 50 psi; turbo gas: 10 psi; desolation temperature: $450{ }^{\circ} \mathrm{C}$; collision activated dissociation gas: 5.0 arbitrary units. The components were monitored from mixture, the temperature was set to $30,40,50$, and $60^{\circ} \mathrm{C}$, respectively.

Capacity factor " $k$ " was calculated from the TR with the following formula:

$$
\mathrm{k}=\left(\mathrm{T}_{\mathrm{R}}-\mathrm{T}_{\mathrm{D}}\right) / \mathrm{T}_{\mathrm{D}}
$$

Where TR is the retention time, TD is dead time, except of the GC MS data obtained from literature (Waters Application) [10]. The $\mathrm{k}$ values were used to compare the behavior of the target compounds under different chromatographic conditions.

\section{Results and discussion}

The first purpose of our research was to choose the target compounds. The selection was based on former studies $[4,10$, 11] and special attention was paid to pick only LC-measurable components, only GC-measureable pesticides and the group that can be determined both way. The selected compounds (depicted in Fig. 2) should have met the following criteria: (i) should be extractable with the citrate buffer of QuEChERS method, ii) should have different $\mathrm{pKo} / \mathrm{w}$ values and (iii) their retention times should be distributed evenly over the entire chromatographic timescale. In the case of GC-measurable components the selections were made based on the date pool of EURL and the Waters application note.

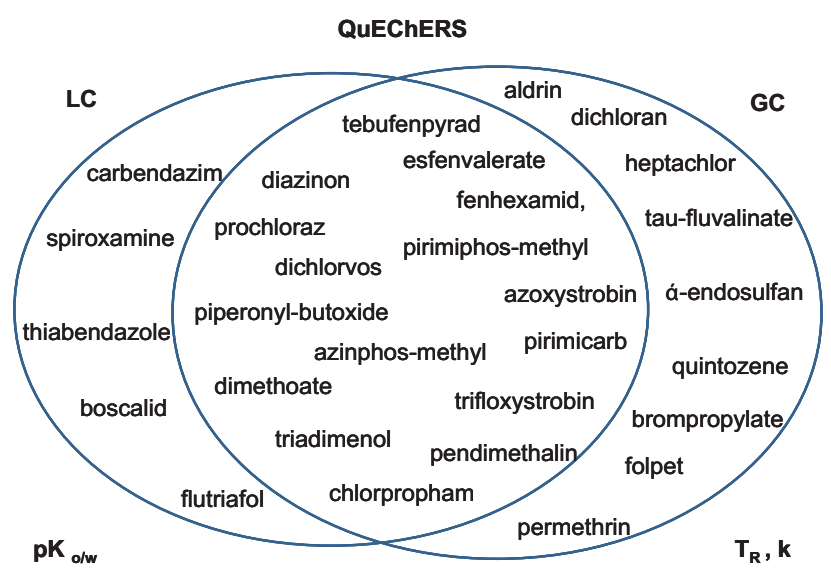

Fig. 2 The 30 choosen compounds

In the first experiment MS method was elaborated. All pesticides were diluted to $1 \mu \mathrm{g} \mathrm{mL}^{-1}$ stock solution and injected individually to the QTRAP 3200 triple quadrupole-linear ion trap mass spectrometer by a syringe. The MS parameters were optimized to these compounds.

\subsection{Results observed with UPC ${ }^{2}$-PDA coupled system}

Originally the a Waters Acquity $\mathrm{UPC}^{2}$ was coupled with a PDA detector that can only detect the target compounds if they are present in huge quantities $10.0 \mu \mathrm{g} \mathrm{mL}^{-1}$, which has a high detection limit that makes this detector type unable to measure real-life samples that are containing the pesticides in lower amount. Therefore it was necessary to use the different systems that could detect different compounds in lower concentration. The UPC ${ }^{2}$-PDA system could detect all 30 compounds except spiroxamine, because at the $\mathrm{pH}$ of the sample this component did not show any UV activity [12].

Molecules present in the Waters GC application as well as measurable by LC-MS were selected. They were ranked based on their capacity factors (Table 1 and 2). These rankings were compared among the different methods, and conclusions about the behavior of the pesticides in supercritical conditions were drawn.

The comparison of capacity factors of runs with methanol (LC) and methanol as co-eluent (UPC ${ }^{2}$ ) showed that azinphosmethyl, diazinon, prochloraz and triadimenol behaved similarly under GC and $\mathrm{UPC}^{2}$ conditions, while chloropham followed the rules of LC chromatography in this new system while the behavior of dichlorvos and piperonil-butoxid showed similarities both that of under LC and GC conditions. X mean no any similarity to between the delaminated sytem retention queue (Table 1).

Table 1 Ranced and compared capacityfactors in the case of $\mathrm{CH}_{3} \mathrm{OH}$

\begin{tabular}{lllll}
\hline $\mathrm{CH}_{3} \mathrm{OH}$ & $\mathrm{LC}$ & $\mathrm{UPC}^{2}$ & $\mathrm{GC}$ & Similarity \\
\hline Azinphos-methyl & 3 & 9 & 9 & $\mathrm{GC}$ \\
Chlorpropham & 5 & 5 & 3 & $\mathrm{LC}$ \\
Diazinon & 8 & 4 & 5 & $\mathrm{GC}$ \\
Dichlorvos & 2 & 1 & 2 & $\mathrm{GC} / \mathrm{LC}$ \\
Dimethoate & 1 & 10 & 4 & $\mathrm{x}$ \\
Permethrin & 11 & 6 & 10 & $\mathrm{x}$ \\
Piperonyl-butoxide & 9 & 7 & 8 & $\mathrm{GC} / \mathrm{LC}$ \\
Pirimiphos-methyl & 7 & 2 & 6 & $\mathrm{x}$ \\
Prochloraz & 6 & 11 & 11 & $\mathrm{GC}$ \\
Tau-fluvalinate & 10 & 3 & 1 & $\mathrm{x}$ \\
Triadimenol & 4 & 8 & 7 & $\mathrm{GC}$ \\
\hline
\end{tabular}

The capacity factors of runs with acetonitrile (LC) and acetonitrile as co-eluent ( $\mathrm{UPC}^{2}$ ) (Table 2) were different from those obtained with methanol (Table 1). Distinctively more components showed similar elution behaviour to GC conditions. Apart from dichlorvos and piperonil-butoxide only permethrin behaved as LC measurable. On the contrary the behaviour of 
dimethoate piromiphos-methyl, and azinphos-methyl showed no similarity neither GC nor LC-like (Table 2)

Table 2 Ranced and compared capacityfactors in the case of $\mathrm{CH}_{3} \mathrm{CN}$

\begin{tabular}{lllll}
\hline $\mathrm{CH}_{3} \mathrm{CN}$ & $\mathrm{LC}$ & $\mathrm{UPC}^{2}$ & $\mathrm{GC}$ & Similarity \\
\hline Azinphos-methyl & 4 & 6 & 9 & $\mathrm{x}$ \\
Chlorpropham & 6 & 2 & 3 & $\mathrm{GC}$ \\
Diazinon & 7 & 5 & 5 & $\mathrm{GC}$ \\
Dichlorvos & 2 & 7 & 2 & $\mathrm{LC} / \mathrm{GC}$ \\
Dimethoate & 1 & 10 & 4 & $\mathrm{x}$ \\
Permethrin & 11 & 3 & 11 & $\mathrm{LC} / \mathrm{GC}$ \\
Piperonyl-butoxide & 9 & 7 & 8 & $\mathrm{LC} / \mathrm{GC}$ \\
Pirimiphos-methyl & 8 & 4 & 6 & $\mathrm{x}$ \\
Prochloraz & 5 & 9 & 10 & $\mathrm{GC}$ \\
Tau-fluvalinate & 10 & 1 & 1 & $\mathrm{GC}$ \\
Triadimenol & 3 & 8 & 7 & $\mathrm{GC}$ \\
\hline
\end{tabular}

\subsection{Results acquired with UPC ${ }^{2}$-ESI-MS and HPLCESI-MS coupled systems}

Tests with the UPC ${ }^{2}$-HPLC-ESI-MS system showed that the number of detectable components depended on the quality of the eluent. Out of the 30 pesticides $\mathrm{CH}_{3} \mathrm{OH}$ allowed the detection of 25 compounds while using $\mathrm{CH}_{3} \mathrm{CN}$ as eluent only 19 pesticides could be detected.

By the LC-MS method all LC-compatible compounds were detected and surprisingly two other, only GC-compatible molecules (namely folpet and alpha- endosulphan). In this method $\mathrm{CH}_{3} \mathrm{CN}$ was the stronger eluent, but in the hyphenated $\mathrm{UPC}^{2}$ system proved to be less effective because less component were measurable. In the Figure 3 the chromatograms of the LC -MS system is depicted. In the case of using LC-MS with $\mathrm{CH}_{3} \mathrm{CN}$ as eluent the peak shapes were Gaussian with less than 0.3 min baseline peak width.

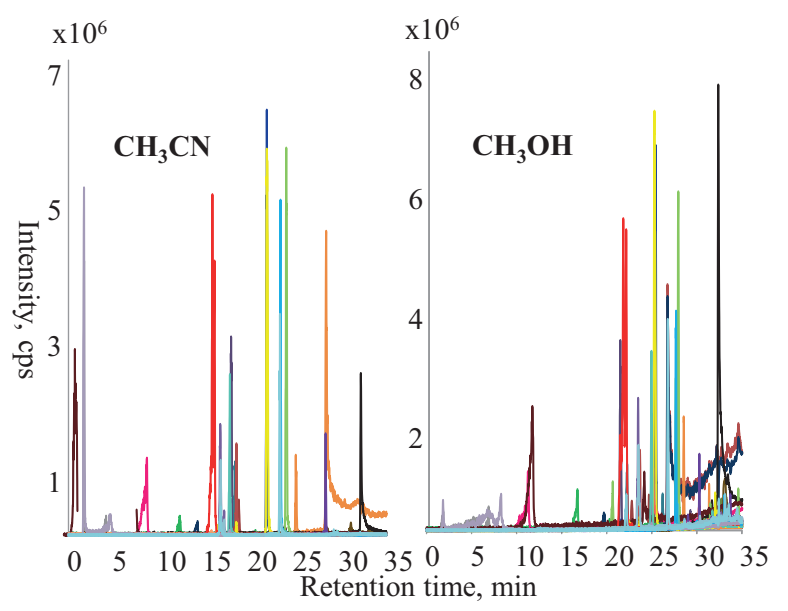

Fig. 3 Elution profiles obtained with the LC-MS system using methanol (left) or acetonitrile (right) as eluent in $40{ }^{\circ} \mathrm{C}$
Figure 4 shows the comparison of UPC ${ }^{2}$-ESI-MS elution profile gained by the use of $\mathrm{CH}_{3} \mathrm{CN}$ and $\mathrm{CH}_{3} \mathrm{OH}$. It is clearly perceptible that by using $\mathrm{CH}_{3} \mathrm{OH}$ containing eluent more components shoved regular peak shape. However it must be mentioned the baseline with this system is not so straight. The reason of this phenomenon is the assistant solvent from the HPLC pump, which was necessary for the ionization of the compounds. Firstly We coupled the UPC ${ }^{2}$ directly to the ESIMS system but in this case there was no ionization observed. Therefore assistant pump was used to the coupling. (Fig. 1) However the assistant solvent increased the ionization of the compunds efficiently, it also elevated baseline.

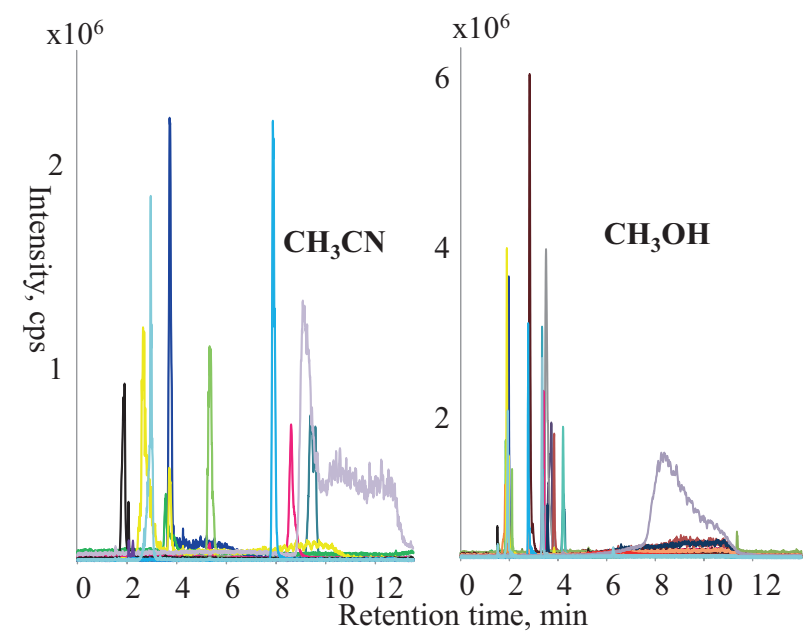

Fig. 4 Elution profiles from the UPC2-ESI-MS system using methanol(left) or acetonitrile (right) as eluent in $40^{\circ} \mathrm{C}$

To handle this problem the UPC ${ }^{2}$ system was coupled successfully either with single quadruple or with ESI TOF MS. The application of these stable coupled systems could result a scientific breakthrough in the field of pesticide analysis.

In general by increasing the temperature the density of the $\mathrm{CO}_{2}$ eluent decreases and so does its solvating ability [13, 14]. Therefore, the higher the temperature the later the components would elute from the column. On the other hand no increase was found in the elution times of dimethoate, tebufenpyrade, prochloraz, piperonyl-butoxide with the addition of $\mathrm{CH}_{3} \mathrm{CN}$ to the eluent. It suggest in the Fig. 5.

\section{Conclusion}

All standards examined can be detected with either PDA or MS methods therefore $\mathrm{UPC}^{2}$ has a potential new applicability in the field of pesticide analytics. The method needs further optimization before it can be applied in routine laboratories. The separation kinetics of the UPC ${ }^{2}$ system does not resemble that either LC or GC therefore it has to be handled as a new, different method. GC is defined by using a gas as its mobile phase and LC is defined by using liquids as its mobile phase, however $\mathrm{UPC}^{2}$ is using both gas and liquid. This convergence 
of mobile phases in combination with a far greater choice of stationary phases makes $\mathrm{UPC}^{2}$ a powerful additional choice for laboratory scientists.

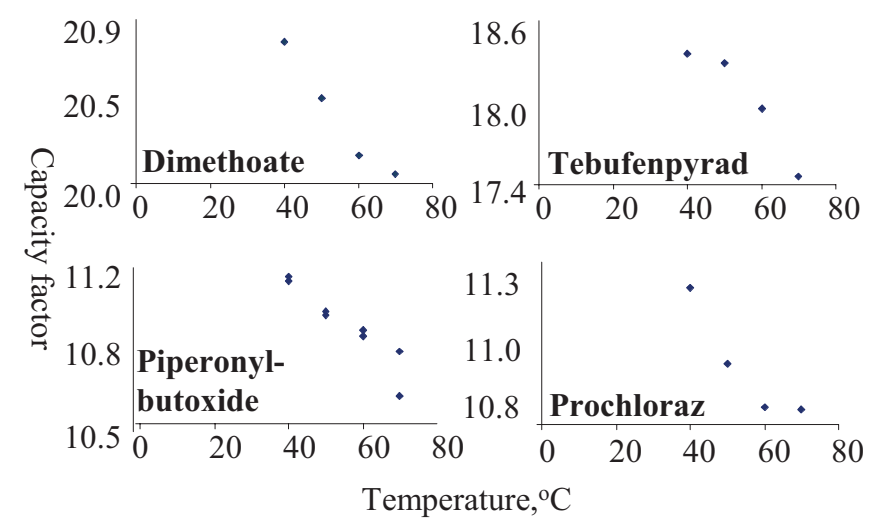

Fig. 5 Elution behaviour in the case of using ancetonitrile coelunent by the examinated $\mathrm{UPC}^{2}$ system

\section{Acknowledgement}

The authors gratefully acknowledge the new demonstration instrument from the Waters Corp. The authors thank the English language proofreading to Gabor Tajti.

\section{References}

[1] Pesticide database. [Online]. Available from: http://ec.europa.eu/food/ plant/pesticides/eu-pesticidesdatabase/public/?event=homepage\&langu age $=\mathrm{EN}$ [Accessed: 25th June 2015]

[2] Hernández, F., Cervera, M. I., Portolés, T., Beltrán, J., Pitarch, E. "The role of GC-MS/MS with triple quadrupole in pesticide residue analysis in food and the environment." Analytical Methods. 5(21), pp. 5875-5894. 2013. DOI: 10.1039/c3ay41104d

[3] Liang, H. C., Bilon, N., Hay, M. T. "Analytical methods for pesticide residues." Water Environment Research. 85(10), pp. 2114-2138. 2013. DOI: $10.2175 / 106143013 X 13698672323227$

[4] Khan, H. A., El-Saeid, M. H. "Chapter 5. Analysis of Pesticides in Food Samples by Supercritical Fluid Chromatography." In: Handbook of pesticides: methods of pesticide residues analysis, (Nollet, L. M. L., Rathore, H S. (ed.)) pp. 93-112, CRC Press, 2010. DOI: 10.1201/9781420082470.ch5

[5] Alder, L., Greulich, K., Kempe, G., Vieth, B. "Residue analysis of 500 high priority pesticides: Better by GC-MS or LC-MS/MS?" Mass Spectrometry Reviews. 25(6), pp. 838-865. 2006. DOI: 10.1002/mas.20091

[6] Saito, M. "History of supercritical fluid chromatography: instrumental development." Journal of Bioscience Bioenginring. 115(6), pp. 590-599. 2013. DOI: 10.1016/j.jbiosc.2012.12.008

[7] Ishibashi, M., Ando, T., Sakai, M., Matsubara, A., Uchikata, T., Fukusaki, E., Bamba, T. "High-throughput simultaneous analysis of pesticides by supercritical fluid chromatography/tandem mass spectrometry." Journal of Chromatography A. 1266, pp. 143-148. 2012.

DOI: $10.1016 /$ j.chroma.2012.09.067

[8] Eaton, S. F., McDonald, P. D. "Waters ACQUITY UPC2 system for convergence chromatography." Chimica Oggi/Chemistry Today. 30(6), pp. 2-3. 2012 .
[9] Ultra Performance Convergence Chromatography. [Online]. Available from: http://www.waters.com/webassets/cms/library/docs/720004225en. pdf [Accessed: 4th May 2015]

[10] Douce, D., Hanock, P., Dudd, S., Mol, H. G. J. "Application of GC-triple quadrupole MS/MS for multi-residue analysis of pesticides in complex matrices." [Online]. Available from: http://www.waters.com/waters/library.htm?cid=511436\&lid=1540588 [Accessed: 4th May 2015]

[11] Kmellár, B., Abrankó, L., Fodora, P., Lehotay, S. J. "Routine approach to qualitatively screening 300 pesticides and quantification of those frequently detected in fruit and vegetables using liquid chromatography tandem mass spectrometry (LC-MS/MS)." Food Additives and Contaminants - Part A Chemistry, Analysis, Control, Exposure and Risk Assessment. 27(10), pp. 1415-1430. 2010. DOI: 10.1080/19440049.2010.490791

[12] European Food Safety Authority (EFSA) "Conclusion of the peer review of the active substance Spiroxamine." EFSA Journal. 8(10), 1719. 2010. URL: http://www.efsa.europa.eu/fr/scdocs/doc/1719.pdf

[13] Kassim, D. M., Zainel, H. A., Al-Asaf, S. A., Talib, E. K. "The temperature dependence of the solubility of carbon dioxide in several extraction solvents." Fluid Phase Equilibria. 1(3), pp. 287-294. 1988. DOI: 10.1016/0378-3812(88)80013-X

[14] Kerlé, D., Ludwig, R., Geiger, A., Paschek, D. "Temperature dependence of the solubility of carbon dioxide in imidazolium-based ionic liquids." Journal of Physical Chemistry B. 113(38), pp. 12727-12735. 2009. DOI: $10.1021 /$ jp9055285 